\title{
Images of Dissipation Layers to Quantify Mixing Within a Turbulent Jet
}

\author{
Douglas A. Feikema, ${ }^{*}$ David Everest, ${ }^{\dagger}$ and James F. Driscoll ${ }^{\ddagger}$ \\ University of Michigan, Ann Arbor, Michigan 48109-2118
}

\begin{abstract}
Images were obtained that visualize the structure of the mixture fraction field and the structure of the scalar dissipation layers that exist near the base of a turbulent jet. To characterize the local mixing rate, measured profiles of the mean dissipation rate were compared with a general scaling analysis. In addition, the joint probability density function $(\zeta, \chi)$ was measured. Some effects of adding coaxial air and swirl are discussed. The especially large values of the mean dissipation rate (up to $30 \mathrm{~s}^{-1}$ ) and the instantaneous dissipation rate (up to $175 \mathrm{~s}^{-1}$ ) that were measured in the base region are due to the large local values of the mean velocity gradient in this region. It was found that the dissipation layers in the base region have a unique structure; the layers tend to be aligned at approximately a 45-deg angle to the flow and thus differ from layers in the far field, which tend to be oriented in an isotropic pattern, as was shown previously. Strong dissipation layers exist at the boundary of the entrained air, and the observed 45-deg alignment in the base region is believed to be due to the ordered vortex pattern in the shear layer. Dissipation layers are typically $0.3 \mathrm{~mm}$ thick. Dissipation rates varied in a manner consistent with the general scaling analysis, and the proper scaling constant was measured to be 9300 .
\end{abstract}

\section{Introduction}

$\mathbf{T}$ HE purpose of the present study is to image the structure of the mixture fraction field and the scalar dissipation layers near the base of a turbulent jet. Previously, Dahm et al. ${ }^{1}$ and Buch et al. ${ }^{2}$ studied the far-field region of a jet at $x / d=39$ and found that the dissipation rate field consists of thin layers in which most of the mixing occurs. Their reported values of mean dissipation rate were as large as $0.08 \mathrm{~s}^{-1}$. In contrast, the present work is concerned with the base region of a jet $(x / d=5$ and 14) where the mean dissipation rates are more than 300 times larger and exceed $30 \mathrm{~s}^{-1}$, as shown later. The near field is important for several practical reasons: the intense mixing in the near field needs to be understood to augment the mixing with geometrical modifications or acoustic methods, and near-field mixing affects the stability of jet flames. Namazian et al. ${ }^{3}$ and Schefer et al. ${ }^{4}$ previously have studied the near-field mixing within a jet; the present conditions differ significantly from those of Ref. 3, because the propane jet described later has a density that is three times larger than the methane jet of Ref. 3 and has a binary diffusion coefficient that is half of that of Ref. 3 . The resulting dissipation rates are significantly different than those of Ref. 3, as expected, and comparisons are presented to determine the validity of theoretical scaling relations. Nandula et $a .^{5}$ recently reported dissipation rates in a reacting jet, which also will be compared with the present results. Some thermal dissipation rates that were determined from temperature measurements have been reported in Refs. 6-9.

The present measurements of the mixture fraction $\zeta$ are used to deduce some profiles of the mean scalar dissipation rate $\chi$, which is defined as $2 D\left(\partial \zeta / \partial x_{i}\right)^{2}$, where $D$ is the diffusion coefficient. Bilger ${ }^{10}$ has shown that $\bar{\chi}$ is an indicator of the local mixing rate, whereas Peters and Williams ${ }^{11}$ have derived a useful scaling relation

Received Jan. 27, 1996; revision received July 18, 1996; accepted for publication July 31, 1996; also published in AIAA Journal on Disc, Volume 2, Number 1. Copyright (C) 1996 by the American Institute of Aeronautics and Astronautics, Inc. All rights reserved.

${ }^{*}$ Research Assistant, Department of Aerospace Engineering; currently Assistant Professor, Department of Mechanical Engineering, University of Alabama, Huntsville, AL 35899. Senior Member AIAA.

${ }^{\dagger}$ Research Assistant, Department of Aerospace Engineering; currently Postdoctoral Fellow, National Institute of Science and Technology, Gaithersburg, MD 20899. Member AIAA.

¥Professor, Department of Aerospace Engineering. Associate Fellow AIAA. that predicts the way in which $\bar{\chi}$ varies in the radial and axial directions within a jet:

$$
\bar{\chi}=c_{1}\left(\rho_{J} / \rho_{A}\right)^{\frac{3}{2}}(U / d)(x / d)^{-4}(r / x)^{2}\left[1+25(r / x)^{2}\right]^{-6}
$$

The global strain rate $(U / d)$ is based on the jet exit velocity $U$ and jet diameter $d ; \rho_{J}$ is the density of the jet fluid prior to mixing, and $\rho_{A}$ is the density of the ambient air prior to mixing. Equation (1) was derived in Ref. 11 by applying the approximation that $\bar{\chi}$ is proportional to $v_{T}(\partial \zeta / \partial r)^{2}$. The turbulent kinematic viscosity $v_{T}$ is proportional to $u_{c l}^{\prime} L$, where centerline velocity fluctuations $u_{c l}^{\prime}$ scale with the mean centerline velocity, which scales with $\left(\rho_{J} / \rho_{A}\right)^{1 / 2} U d / x$. Since the integral scale $L$ is proportional to $x$ in a jet, Ref. 11 employs a value of $v_{T}$ of $\left(\rho_{J} / \rho_{A}\right)^{1 / 2} U d / 70$. The quantity $\partial \zeta / \partial r$ is determined by differentiating the established relation for $\zeta$ in a jet, ${ }^{11}$ which is $6(x / d)^{-1}\left(\rho_{J} / \rho_{A}\right)^{1 / 2}\left[1+25(r / x)^{2}\right]^{-2}$.

Equation (1) predicts that mean dissipation rate should scale with the global strain rate $U / d$ and should be zero on centerline. Dissipation rate is predicted to be maximum at a radial location of $0.09 x$ and should be maximum near the base of the jet and decrease in the axial direction due to the $x^{-4}$ factor. As predicted by Eq. (1), the mixing rates are expected to decrease in the $x$ direction as the mean gradients (and the amount of jet fluid left to mix) decrease to zero.

\section{Experimental Apparatus}

A turbulent jet was created by flowing gaseous propane vertically upward at a velocity of $20.6 \mathrm{~m} / \mathrm{s}$ through a fuel tube having an inside diameter $d$ of $4.8 \mathrm{~mm}$. The jet Reynolds number is $1 \times 10^{4}$, and the global strain rate $U / d$ is $4,290 \mathrm{~s}^{-1}$. The fuel tube exit conditions can be considered to be those of fully developed pipe flow because the fuel tube is a straight tube having a length-to-diameter ratio of 104. To eliminate dust particles that can affect the laser diagnostics, the jet is surrounded by a coflowing airstream that has a diameter of $120 \mathrm{~mm}$ and has a sufficiently low velocity $(0.2 \mathrm{~m} / \mathrm{s})$ such that the coflowing air does not affect the near field of the jet. In addition to the simple jet case, the case of a jet with coaxial air was considered by inserting a 31.4-mm-diam coaxial air tube that surrounds the central fuel jet. The coaxial air velocity is $1.45 \mathrm{~m} / \mathrm{s}$. A third case that was considered was that of a jet with swirl added to the coaxial air; the coaxial air axial velocity was set to $2.9 \mathrm{~m} / \mathrm{s}$ and the swirl number of the coaxial air was set to 0.89 by introducing some air in the tangential direction upstream of the jet exit in a manner described in Ref. 12. Swirl number is defined as the ratio of angular momentum to the axial momentum of the coaxial airflow; Ref. 12 shows that the swirl number for the present geometry is $\pi r_{0} R A_{t}^{-1}\left[m_{\theta} /\left(m_{\theta}+m_{A}\right)\right]^{2}$, 
where $r_{0}$ is the coaxial air tube radius and $R$ is the radius, where tangential air having measured mass flow rate $m_{\theta}$ is introduced, $A_{t}$ is the area of the tangential air inlets, and $m_{A}$ is the measured mass flow rate of air entering the swirl chamber.

Images of the mixture fraction field were obtained with the planar Rayleigh scattering system that is described in Ref. 6. A Candela EDL-8 flashlamp-pumped dye laser and the optics described later provided a laser light sheet having a duration of $1.5 \mu \mathrm{s}$, a wavelength of $514 \mathrm{~nm}$, and an $e^{-2}$ width of $170 \mu$. Background light was minimized by passing the light sheet through two consecutive entrance slits and an exit slit. At each jet location selected, 200 images were collected using a cooled, intensified charged-coupled device (CCD) array camera. A $0.9 \times 0.9 \mathrm{~cm}$ spatial region was imaged on a $576 \times 384$ pixel array using 1:1 optics. Pixel dimensions are $23 \times 23 \mu$. Images were stored on a 687 Mbyte disk of a $486 / 33$ $\mathrm{MHz}$ computer.

\section{Data Analysis}

The mixture fraction $\zeta$ is equal to the mass fraction of jet fluid at each location in this binary component mixing process; $\zeta$ is determined from the Rayleigh scattered light intensity $I$ as follows. Scattered light intensity $I$ is related to the mole fractions of jet fluid $X_{J}$ by

$$
I=C_{1}\left[X_{J} \sigma_{J}+\left(1-X_{J}\right) \sigma_{A}\right]
$$

where $C_{1}$ is a constant; $\sigma_{J}$ and $\sigma_{A}$ are the known Rayleigh scattering differential cross sections of the jet fluid and the air. Since $\zeta$ equals the local mass fraction of jet fluid, it is related to the mole fraction of jet fluid by

$$
\zeta=\frac{M_{J} X_{J}}{M_{J} X_{J}+M_{A}\left(1-X_{J}\right)}
$$

where $M_{A}$ and $M_{J}$ are the molecular weights of air and jet fluid. Combining Eqs. (2) and (3) yields

$$
\zeta=\frac{\left(I^{\prime}-s\right)}{A+B\left(I^{\prime}-s\right)}
$$

The quantity $I^{\prime}$ is the Rayleigh scattered light intensity normalized by the value that is measured at $x=0, r=0$, where $\zeta$ is unity (pure jet fluid); $m$ is the known ratio of molecular weights $\left(M_{A} / M_{J}\right)$ and $s$ is the known ratio of scattering cross sections $\left(\sigma_{A} / \sigma_{J}\right)$. Constants $A$ and $B$ are $m(1-s)$ and $(1-m)$, respectively. If pure jet fluid is present, $I^{\prime}$ equals 1 and $\mathrm{Eq}$. (4) indicates that $\zeta$ will be unity. If pure air is present in the measuring volume, $I^{\prime}$ approaches the value of $s$, and the right-hand side of Eq. (4) approaches zero. Although Eq. (4) is nonlinear, it deviates by less than $10 \%$ from a linear relation between $\zeta$ and $I^{\prime}$, and so there are no situations for which the measuring technique becomes less sensitive due to nonlinearities.

The scalar dissipation rate in cylindrical coordinates is defined as

$$
\chi=2 D\left[\left(\frac{\partial \zeta}{\partial x}\right)^{2}+\left(\frac{\partial \zeta}{\partial r}\right)^{2}+\left(\frac{r^{-1} \partial \zeta}{\partial \theta}\right)^{2}\right]
$$

where $D$ is the binary diffusivity of propane into air at $300 \mathrm{~K}$, which is $0.11 \mathrm{~cm}^{2} / \mathrm{s}$. The third term in Eq. (5) cannot be measured from the present planar images but was set equal to the second term. This approximation would be exact if the turbulence was isotropic; in a jet flow it should introduce relatively small errors when time averages are computed because the surface on which the mixture fraction equals a given value will be highly contorted at the jet Reynolds number of $1 \times 10^{4}$; therefore the normal to any given (wrinkled) contour surface will be as likely to point in the azimuthal direction as in the radial direction. Previous measurements of temperature in a heated jet were made by Antonia et al., ${ }^{13}$ who reported that the mean values of $(\Delta T / \Delta y)^{2}$ and $(\Delta T / \Delta z)^{2}$ are nearly equal. Sreenivasan et al. ${ }^{14}$ also showed that the probability density functions (PDFs) and spectra of $(\Delta T / \Delta y)^{2}$ and $(\Delta T / \Delta z)^{2}$ are similar. A digital filter was used that assigns the median value of a $5 \times 5$ pixel region $(115 \times 115 \mu \mathrm{m})$ to the central pixel. The derivatives $\partial \zeta / \partial x$ and $\partial \zeta / \partial y$ then were determined by using the following 15 -point central differencing scheme ${ }^{15}$ :

$$
\begin{aligned}
\frac{\chi_{i j}}{2 D} & =\left[\frac{-2 \zeta_{i, j-2}+8 \zeta_{i, j-1}-8 \zeta_{i, j+1}+2 \zeta_{i, j+2}}{48 \Delta}\right. \\
& +\frac{-2 \zeta_{i+2, j-2}+8 \zeta_{i+1, j-1}-8 \zeta_{i-1, j+1}+2 \zeta_{i-2, j+2}}{96 \Delta} \\
& \left.+\frac{-2 \zeta_{i-2, j-2}+8 \zeta_{i-1, j-1}-8 \zeta_{i+1, j+1}+2 \zeta_{i+2, j+2}}{96 \Delta}\right]^{2} \\
& +\left[\frac{-2 \zeta_{i+2, j}+8 \zeta_{i-1, j-1}-8 \zeta_{i-1, j}+2 \zeta_{i-2, j}}{48 \Delta}\right. \\
& +\frac{-2 \zeta_{i+2, j-2}+8 \zeta_{i+1, j-1}-8 \zeta_{i-1, j+1}+2 \zeta_{i-2, j+2}}{96 \Delta} \\
& \left.+\frac{-2 \zeta_{i-2, j-2}+8 \zeta_{i-1, j-1}-8 \zeta_{i+1, j+1}+2 \zeta_{i+2, j+2}}{96 \Delta}\right]^{2}
\end{aligned}
$$

\section{Spatial Resolution Requirements}

Several modifications were made to achieve the $170-\mu \mathrm{m}$ laser light sheet thickness: the laser beam was expanded to three times its normal diameter before final focusing, apertures were added that blocked $50 \%$ of the light but helped to reduce the sheet thickness, a three-prism tuner was added to reduce the laser bandwidth to $2 \AA$, and the CCD array camera was used to directly measure the laser beam waist diameter. The laser beam first passed through two spherical lens (50- and 150-mm focal lengths) to expand the beam from 6 to $18 \mathrm{~mm}$. Beam focusing principles indicate that by tripling the incident beam diameter the beam waist diameter should be significantly reduced. The expanded beam then passed through a circular aperture that was adjusted to block the outer portions of the beam that did not focus properly. A cylindrical lens (150-mm focal length) was used to form the light sheet. Two slits were used to prevent background light (reflected off the lenses) from entering the test section; the slits also helped to reduce the light sheet thickness. The apertures and slits blocked about $50 \%$ of the laser light; the laser output was $400 \mathrm{~mJ}$ per pulse and the useful energy in the sheet was $200 \mathrm{~mJ}$ per pulse. A CCD array camera was used to measure the actual sheet thickness; the cylindrical lens was replaced with a biconvex lens of the same focal length $(150 \mathrm{~mm})$ and the Rayleigh scattering from room air was imaged; the resulting $e^{-1}$ waist diameter was measured to be $120 \mu \mathrm{m}$, and the corresponding $e^{-2}$ waist diameter is $170 \mu \mathrm{m}$ at the edge of the $0.9 \times 0.9 \mathrm{~cm}$ imaging region.

To measure scalar gradients using Eqs. (5) and (6), it is necessary to resolve the smallest scale of the scalar fluctuations, which is denoted the strain-limited molecular diffusion scale ${ }^{1}\left(\lambda_{D}\right)$. Values of $\lambda_{D}$ were measured by Dahm et al. ${ }^{1}$ and were found to equal

$$
\lambda_{D}=25 \delta\left[U_{c l} \delta / \nu\right]^{-\frac{3}{4}} S c^{-\frac{1}{2}}
$$

where $U_{c l}$ is the jet centerline velocity and $\delta$ is defined in Ref. 1 as the jet full width between locations where the velocity is 0.2 times the centerline velocity; with this definition, $\delta$ is shown in Ref. 1 to equal $0.44 x$. In the present work, the Schmidt number is 0.7 and the kinematic viscosity of air $\left(0.15 \mathrm{~cm}^{2} / \mathrm{s}\right)$ is used in Eq. (7) because the dissipation layers are strongest near the air boundary. The term $U_{c l}$ in Eq. (7) can be replaced by $c_{2}\left(J / \rho_{c l}\right)^{1 / 2} x^{-1}$ for axisymmetric turbulent jets ${ }^{16}$ and the momentum flux $J$ is replaced by $c_{3} \rho_{J} U_{J}^{2} d^{2}$, where $\rho_{J}$ and $U_{J}$ are the density and velocity at the jet exit. Wygnanski and Fiedler ${ }^{17}$ determined the constant $c_{2}$ and $c_{3}$ and showed that $U_{c l}$ equals $5.2 U_{J}\left(\rho_{J} / \rho_{A}\right)^{1 / 2}(d / x)$. Inserting this value of $U_{c l}$ and the preceding value of $\delta(=0.44 x)$ into Eq. (7), the smallest dissipation layer thickness that must be resolved is

$$
\lambda_{D}=(5.9 x)\left(U_{J} d / v_{A}\right)^{-\frac{3}{4}}\left(\rho_{J} / \rho_{c l}\right)^{-\frac{3}{8}} S c^{-\frac{1}{2}}
$$

For the present conditions, Eq. (8) yields a minimum dissipation layer thickness $\left(\lambda_{D}\right)$ of $590 \mu \mathrm{m}$ at $x / d=14$, whereas $\lambda_{D}$ is $210 \mu \mathrm{m}$ at $x / d=5$. The measured light sheet thickness at the $e^{-2}$ intensity points is described earlier to be $170 \mu \mathrm{m}$. 
The amount of error associated with the spatial resolution was estimated by calculating the area under the dissipation energy spectrum that was not resolved. The scalar spectrum reported for temperature fluctuations in Ref. 10 was used. Using this approach the actual values of $\bar{\chi}$ are estimated to be 1.4 times larger than the measurements at $x / d=5$ and 1.1 times larger than measurements at $x / d=14$. Namazian et al. ${ }^{3}$ performed a similar calculation, and for their larger value of sheet thickness $(220 \mu \mathrm{m})$, they estimated that actual values of $\bar{\chi}$ are 1.6 times larger than the measured values, which is consistent with the present estimates. Because of the uncertainty of such an estimate, no corrections were made to the present data.

\section{Results}

A. Images of Mixture Fraction and Scalar Dissipation Rates

Some images of the mixture fraction field for the jet case (i.e., no coaxial air or swirl) are presented in Fig. 1. The upper two rows of images in Fig. 1 were obtained at $x / d=14$, whereas the lower two rows were obtained at $x / d=5$. The jet centerline is located on the right side of each $0.9 \times 0.9 \mathrm{~cm}$ image. Each one of the images of mixture fraction $(a-h)$ in Fig. 1 was obtained at a different time and is independent of the other images. A gray scale is used to represent mixture fraction; white denotes pure air $(\zeta=0)$, whereas black denotes pure jet fluid $(\zeta=1)$. In addition, the region near the jet edge where $\zeta$ is in the range between 0.03 and 0.14 is represented as a black region. This outer black region could be identified as a flammable layer since within this layer the propane and air have mixed sufficiently to create a mixture that lies within the flammability limits for propane and air. The white line that is seen in Fig. 1b occurs where mixture fraction is 0.4 ; this line illustrates that the structure of the air boundary $(\zeta=0.03)$ and the $\zeta=0.4$ boundary are similar. In case 1f, for example, a second white line is plotted where $\zeta=0.75$, but this contour is not present in all images because all of the fuid in the image may be mixed to $\zeta$ values less than 0.75 .

Some evidence of vortex-induced structure is evident in Fig. 1. The mushroom-shaped structures in Figs. 1a and 1e appear to be due to vortices that are moving in the radial direction. Note that the peninsula-shaped white regions of entrained air in Figs. $1 \mathrm{~b}$ and $1 \mathrm{~d}$ are aligned along a line that is inclined at approximately $45 \mathrm{deg}$ to the flow, as shown by the line in the schematic in Fig. 1. Similarly, the strongest scalar dissipation layers shown in the right column in Fig. 1 also are inclined at approximately $45 \mathrm{deg}$ to the flow. The inclination of the peninsulas of entrained air is explained by the
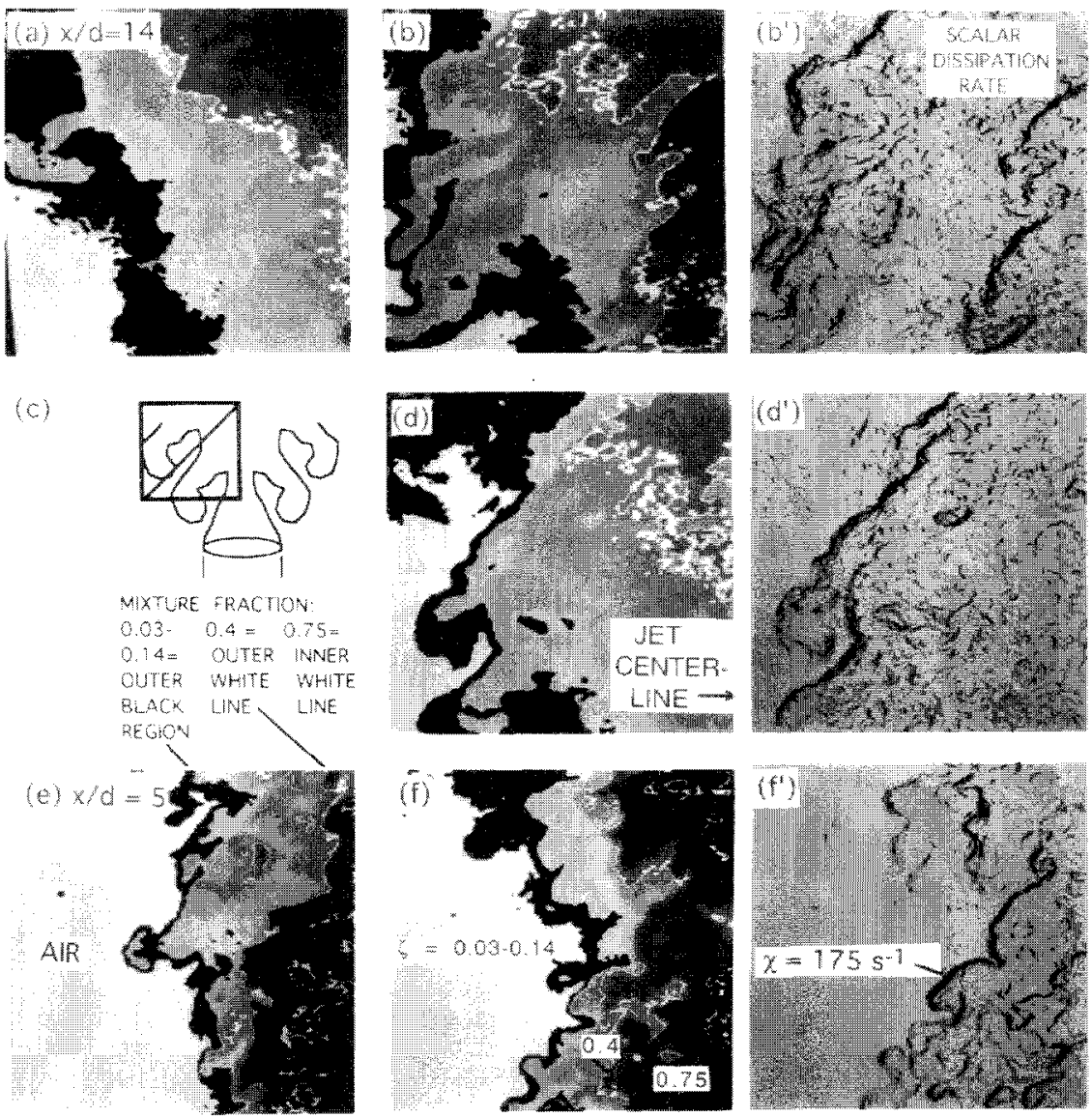

(i)
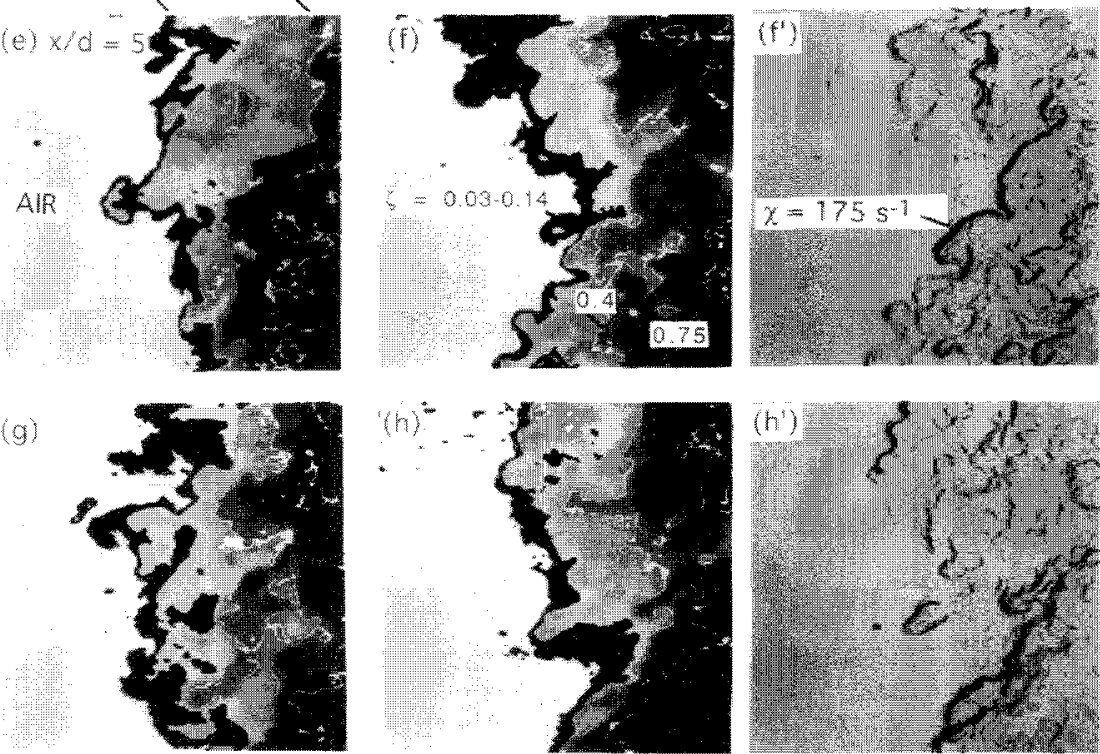

Fig. 1 Instantaneous mixture fraction $\zeta$ and scalar dissipation rate $\chi$ for the jet case. No coaxial air or swirl. Right side of image is jet centerline; field of view is $0.9 \times 0.9 \mathrm{~cm}$. Top two rows: $x / d=14$; bottom two rows: $x / d=5$. Images $b^{\prime}, d^{\prime}, f^{\prime}$, and $h^{\prime}$ represent scalar dissipation rates corresponding to images $b, d, f$, and $h$, respectively. The edge of the jet $(\zeta=0.03-0.14)$ is shown as a wrinkled black layer; proceeding to the right is a light gray region $(\zeta=0.14-0.4)$ followed by a white line marking the $\zeta=0.4$ contour. A second white line marks the $\zeta=0.75$ contour, but this $\zeta$ value occurs only in the lower two rows. The black region on the right represents $\zeta=0.75-1.0$. Strongest dissipation layers are aligned at approximately 45 deg to the jet axis. 
counterclockwise rotation of vortices in the region of interest. This rotation tends to engulf air radially inward at a location upstream of each vortex; the rotation then convects the air downstream (i.e., upward in Fig. 1), as is shown in the schematic in Fig. 1. The most intense mixing occurs at the boundary of these inclined regions of engulfed air, causing the dissipation layers to be inclined as shown.

The most intense scalar dissipation layers in Fig. 1 have a maximum value of $\chi$ of $175 \mathrm{~s}^{-1}$ and have a layer thickness that is typically $0.2 \mathrm{~mm}$. These two values are consistent because if the mixture fraction was to vary by a typical amount of $\Delta \zeta=0.5$ over a distance of $\Delta r=0.2 \mathrm{~mm}$ near the entrained air boundary, the resulting dissipation rate of $2 D(\Delta \zeta / \Delta x)^{2}$ would be $140 \mathrm{~s}^{-1}$. The most intense dissipation layers only occupy a small volume of the flowfield, which is similar to the far-field findings of Dahm et al. ${ }^{1}$ and Buch et al. ${ }^{2}$ The maximum time-averaged dissipation rate of $15 \mathrm{~s}^{-1}$ in the jet (that is deduced later) thus is significantly less than the maximum instantaneous value of $175 \mathrm{~s}^{-1}$.

\section{B. Profiles of Mean Scalar Dissipation Rates $\bar{\chi}$}

Some radial profiles of $\bar{\chi}$ measured in the jet are shown in Fig. 2. Each data point plotted represents the average of 5000 values that are contained in a $5 \times 5$ pixel region within 200 images. Figure $2 b$ shows that $\bar{\chi}$ reaches a maximum value, denoted $\bar{\chi}_{\max }$, near the mean boundary of the jet at $r / x=0.09$. Figure 2 also shows that $\bar{\chi}_{\max }$ decreases in the axial direction from $14.2 \mathrm{~s}^{-1}$ at $x / d=5$ to $5.0 \mathrm{~s}^{-1}$ at $x / d=14$.

Figure $2 \mathrm{a}$ is a comparison of the shape of the predicted profile of mean dissipation rate [Eq. (1)] with the present measurements at $x / d=14$. Equation (1) predicts only the shape of the $\bar{\chi}$ profile and not the absolute magnitude of $\bar{\chi}$ because the scaling constant $c_{1}$ in Eq. (1) must be determined from measurements; a value of $c_{1}=9300$ was found to provide best agreement between the solid curve in Fig. $2 \mathrm{a}$ and the data.

Some comparisons of dissipation rates that were measured in three studies are listed in Table 1. The first column in Table 1 lists values of $\bar{\chi}_{\max }$, the peak value of $\bar{\chi}$ at the $x / d$ location listed in

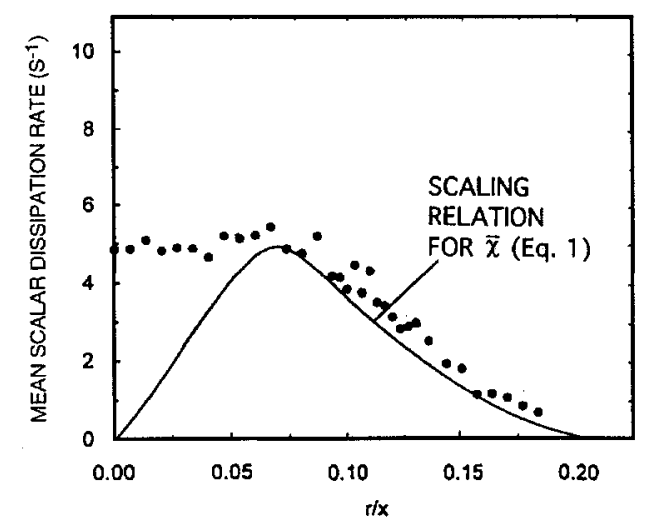

a) $x / d=14$

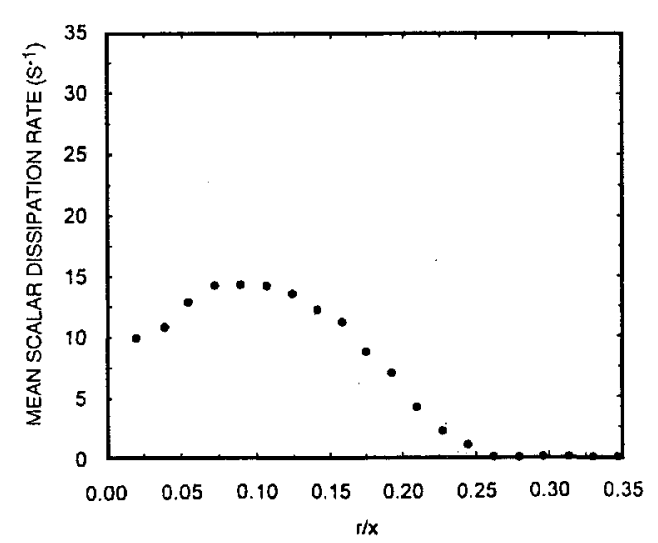

b) $x / d=5$

Fig. 2 Mean scalar dissipation rate $(\bar{\chi})$ measured in the jet compared to the scaling relation of Peters and Williams [Eq. (1)]. For best fit, the constant $c_{1}$ in Eq. (1) is 9300.
Table 1 Comparison of scalar dissipation rates reported in several studies

\begin{tabular}{|c|c|c|c|c|c|}
\hline & $\begin{array}{l}\bar{\chi} \max \\
\mathbf{s}^{-1}\end{array}$ & $\rho_{J} / \rho_{A}$ & $\begin{array}{c}U / d, \\
\mathrm{~s}^{-1}\end{array}$ & $x / d$ & $\begin{array}{l}\text { Scaling constant }\left(c_{1}\right) \\
\text { in Eq. (1) }\end{array}$ \\
\hline $\begin{array}{l}\text { Present study } \\
\text { (propane jet) }\end{array}$ & $\begin{array}{r}14.2 \\
5.2\end{array}$ & $\begin{array}{l}1.52 \\
1.52\end{array}$ & $\begin{array}{l}4290 \\
4290\end{array}$ & $\begin{array}{r}5 \\
14\end{array}$ & $\overline{9300}^{\mathrm{a}}$ \\
\hline $\begin{array}{l}\text { Effelsburg and Peters }{ }^{18} \\
\text { (propane jet) }\end{array}$ & $\begin{array}{l}3.0 \\
1.45\end{array}$ & $\begin{array}{l}1.52 \\
1.52\end{array}$ & $\begin{array}{l}6670 \\
6670\end{array}$ & $\begin{array}{r}5 \\
21\end{array}$ & $\overline{8500}^{\mathrm{a}}$ \\
\hline $\begin{array}{l}\text { Namazian et al. }{ }^{3} \\
\text { (methane jet) }\end{array}$ & $\begin{array}{l}2.9 \\
0.5\end{array}$ & $\begin{array}{l}0.55 \\
0.55\end{array}$ & $\begin{array}{l}3900 \\
3900\end{array}$ & $\begin{array}{r}5 \\
17\end{array}$ & $\overline{9800}^{\mathrm{a}}$ \\
\hline $\begin{array}{l}K-\varepsilon \text { model }^{18} \\
\text { (propane jet) }\end{array}$ & 0.1 & 1.52 & 6670 & 38 & 6300 \\
\hline
\end{tabular}

${ }^{\mathrm{a}}$ The constant $c_{1}$ was not determined at $x / d=5$ because this is in the jet core region where Eq. (1) is not valid.

column 4. To compare the results, the scaling constant $c_{1}$ in Eq. (1) was determined for each study by dividing $\bar{\chi}_{\max }$ by all of the factors on the right side of Eq. (1) (except $c_{1}$ ) and the resulting values of $c_{1}$ are shown in the last column of Table 1 . Only values of $x / d=14$ or larger were considered. The present results indicate that $c_{1}$ is 9300 , whereas the results of Refs. 3 and 18 yield a scaling constant $c_{1}$ of 8500 and 9800 , respectively. These differences are within the experimental uncertainty of the present types of measurements. It can be concluded that Eq. (1) appears to yield a reasonable estimate of $\bar{\chi}$ over a wide range of conditions for turbulent jets.

Recently Nandula et al. ${ }^{5}$ have reported dissipation rates measured in a hydrogen jet flame. Comparisons of their findings with the nonreacting jets in Table 1 require caution because a flame can create intense scalar gradients near the reaction zone yet can reduce the gradients by lowering the Reynolds number in other regions. Nandula et al. measure a value of $15 \mathrm{~s}^{-1}$ for the radial term contribution to $\bar{\chi}_{\max }$, and thus an estimate of their value of $\bar{\chi}_{\max }$ is $45 \mathrm{~s}^{-1}$ when the axial and azimuthal contributions in Eq. (5) are considered. Values of $U / d, x / d$, and $\rho_{J} / \rho_{A}$ were $250,000 \mathrm{~s}^{-1}, 9$, and 0.069 , yielding a value of the constant $c_{1}$ in Eq. (1) of 24,000 for their flame. Therefore the dissipation rates reported by Nandula et al. ${ }^{5}$ are 2.5 times larger than the nonreacting hydrocarbon jet results reported herein and in Refs. 3 and 18 . The differences may be due to changes caused by a flame or the large differences between the density and diffusitivity of hydrogen and propane, which may not be correctly scaled by Eq. (1). An important research question is the extent to which chemical reactions affect the local mixing rates and values of $\bar{\chi}$ and whether these effects are correctly predicted by statistical models. ${ }^{18}$

\section{Effects of Coaxial Air and Coaxial Air with Swirl}

A significant enhancement of the overall mixing rate was achieved by surrounding the central jet with a 31.4-mm-diam stream of coaxial air in one case and with coaxial air with swirl in another case. The axial air velocity was $1.45 \mathrm{~m} / \mathrm{s}$ for the coaxial air case and was $2.9 \mathrm{~m} / \mathrm{s}$ for the swirl case (swirl number $=0.89$ ). Figure 3 shows that coaxial air reduces the mean mixture fraction on centerline at $x / d=14$ to a value of 0.26 , which is half of the value in the jet with no coaxial air $(0.53)$. Thus the overall mixing rates are significantly larger in the coaxial air and swirl air cases than in the jet case. Note that Fig. 3b shows that no significant differences exist between the three cases at $x / d=5$, and so the differences in the mixing rates must occur between $x / d$ locations of 5 and 14 .

The structure of the jet flow with coaxial air is shown in the upper two rows of images in Fig. 4. Images in the upper row (Figs. 4a and $4 \mathrm{~b}$ ) were obtained at $x / d=14$; note that there is no unmixed jet fluid (which is black) on centerline, which indicates that the flow is relatively well mixed. The jet edge in these cases is highly contorted. The second row of images (Figs. $4 \mathrm{c}$ and $4 \mathrm{~d}$ ) were obtained at $x / d=5$ where unmixed jet fluid (black) does exist and where the jet edge is less contorted than at $x / d=14$. At both locations the most intense dissipation layers are aligned at approximately 45 deg to the flow direction, as was observed in the jet with no coaxial air. This inclination angle was estimated by visual inspection only and not by statistical analysis.

The most severe fragmentation of the central jet fluid occurs when swirl is added to the coaxial air, as is shown by the lower two rows of images in Fig. 4. Images in the third row (Figs. 4e and $4 \mathrm{f}$ ) were obtained at $x / d=14$ in the swirl flow where mixing is especially 

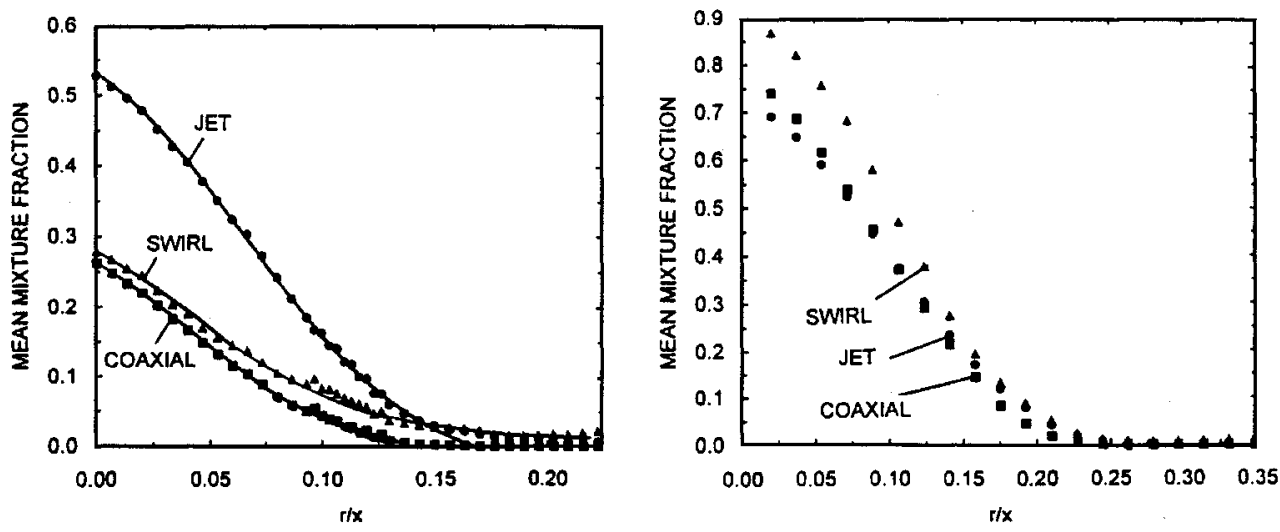

a) $x / d=14$

b) $x / d=5$

Fig. 3 Enhanced overall mixing caused by the addition of coaxial air (or coaxial air with swirl) to a jet, as deduced from a decrease in centerline mean mixture fraction at $x / d=14$. Note that at $x / d=5$ there is little enhancement of mixing that is yet to develop.
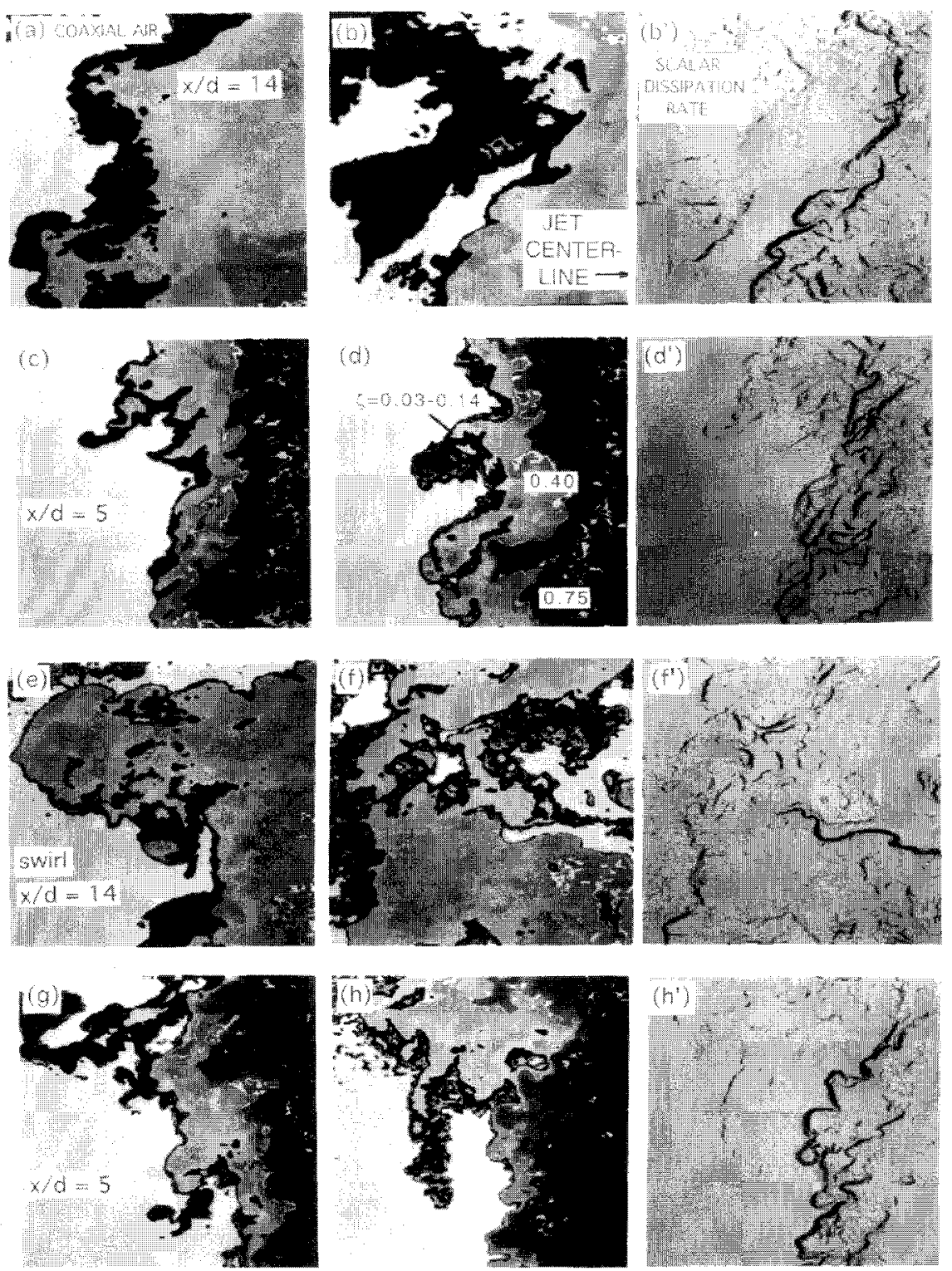

Fig. 4 Mixture fraction and scalar dissipation rate for the jet with coaxial air (top two rows) and for the jet with coaxial air with swirl (bottom two rows). Right side of image is jet centerline; field of view is $0.9 \times 0.9 \mathrm{~cm}$. Top row $\left(a-b^{\prime}\right)$ : coaxial air, $x / d=14$; second row $\left(c-d^{\prime}\right)$ : coaxial air, $x / d=5$; third row $\left(e-f^{\prime}\right)$ : swirl air, $x / d=14$; bottom row $\left(f-h^{\prime}\right)$ : swirl air, $x / d=5$. Images $b^{\prime}, d^{\prime}, f^{\prime}$, and $h^{\prime}$ represent dissipation rates corresponding to images $b, d, f$, and $h$, respectively. Leftmost black region: $\zeta=0.03-0.14$, outer white line: $\zeta=0.40$, inner white line: $\zeta=0.75$. Strongest dissipation layers in Figs. $b^{\prime}, d^{\prime}, f^{\prime}$ and $h^{\prime}$ are aligned at approximately 45 deg to the jet axis. 
intense; there is little unmixed jet fluid (black) observed on centerline. Figure $4 \mathrm{e}$ shows a large peninsula region of partially mixed fluid that appears to be convected radially outward. A violent fragmentation of the mixture fraction field appears in Fig. 4f, in which islands of pure ambient air are entrained to the jet centerline, which exists on the right side of the image. The most intense dissipation layers in Fig. $4 f^{\prime}$ occur at the boundary of the entrained air islands. Severe fragmentation of the jet edge also is noted at $x / d=5$ (Figs. $4 \mathrm{~g}$ and $4 \mathrm{~h}$ ). It can be concluded that, based on Figs. 3 and 4 , coaxial air and coaxial air with swirl tend to produce more surface area for mixing to occur than does a simple jet. Figure 4 displays larger entrainment patterns, more fragmentation, and larger islands of entrained air than Fig. 3. Furthermore, swirl causes the jet fluid to move radially outward and thus occupy a larger volume in space, allowing air to penetrate and mix more effectively.

The addition of coaxial air with swirl causes a significant increase in the mean dissipation rate, as shown by Fig. 5. Figure 5a shows that $\bar{\chi}_{\max }$ doubles (from 5.2 to $12 \mathrm{~s}^{-1}$ at $x / d=14$ and from 14.2 to $30 \mathrm{~s}^{-1}$ at $x / d=5$ ) as coaxial air with swirl is added. This $\bar{\chi}_{\max }$ value of 30 $\mathrm{s}^{-1}$ is larger than any previously reported value and is an indicator of an intense mixing process. This large value of $\bar{\chi}_{\max }$ is consistent with the images described earlier; for this case Fig. $4 \mathrm{f}$ shows that many fragmented islands of entrained air occur and each island contributes a boundary along which a dissipation layer is formed. Note that the maximum instantaneous value of $\chi$ that is typical of any one layer in Fig. 4 is $175 \mathrm{~s}^{-1}$, and so the instantaneous dissipation rate in each layer is not significantly larger than that found in the corresponding jet with no coaxial air; instead the larger values of $\bar{\chi}$ with coaxial air and swirl are primarly due to the increased number of layers per unit volume, which results from the entrainment of large numbers of islands of air. The swirl that is applied in the present work is not sufficient to cause internal recirculation, but it clearly broadens the jet and creates centrifugal forces that help to entrain islands of air.

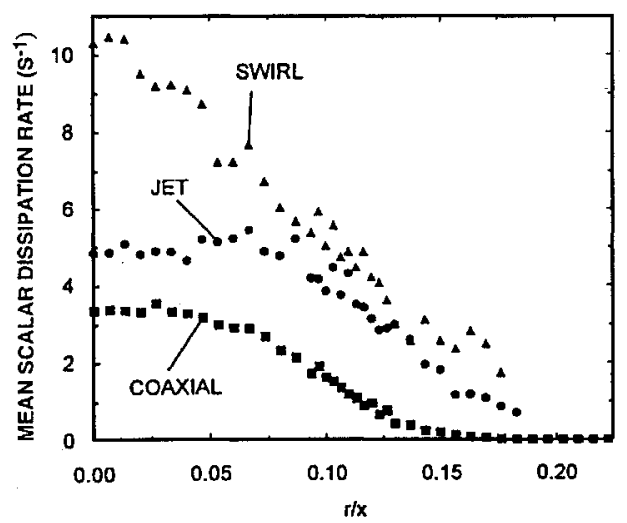

a) $x / d=14$

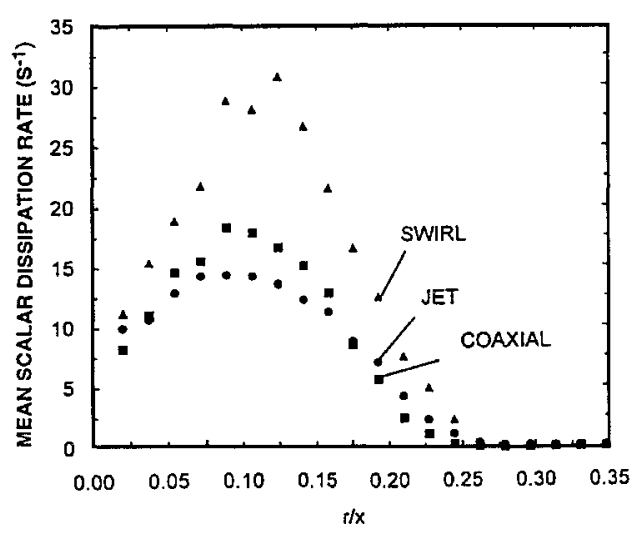

b) $x / d=5$

Fig. 5 Mean scalar dissipation rates $(\bar{\chi})$, which quantify local mixing rates, compared for three cases. In all cases, the propane jet velocity is $20.6 \mathrm{~m} / \mathrm{s}$ and the jet diameter is $4.8 \mathrm{~mm}$.
When coaxial air alone (with no swirl) is added to the jet, Fig. 5a shows that the mean dissipation rates are less than those of the jet case at $x / d=14$. This may appear to contradict the fact that the coaxial air case experiences the most rapid overall mixing rate, as was evidenced by the low value of centerline mean mixture fraction in Fig. 3 for the coaxial air case. However, there is no contradiction; because of enhanced mixing due to coaxial air, the fluid already is fairly well mixed at $x / d=14$ and there is relatively less fluid left to mix. Therefore the local dissipation rate at $x / d=14$ for the coaxial air case is less than that of a pure jet. Figures $4 a$ and $4 b$ show that there is no fluid with mixture fraction above 0.4 (i.e., fluid that is shaded black) even on the centerline at $x / d=14$ with coaxial air. Thus the relatively well-mixed fluid in this case will no longer experience large values of mixture fraction gradients.

One useful measure of the degree of unmixedness in the jet is the rms fluctuation of the mixture fraction $\left(\zeta_{\mathrm{rms}}^{\prime}\right)$, which is plotted in Fig. 6. Note that $\zeta_{\mathrm{rms}}^{\prime}$ is small at locations where the fluid is fully mixed and at locations where the fluid has not yet begun to mix. Figure 6 shows that values of $\zeta_{\mathrm{mm}}^{\prime}$ follow the same trends as the dissipation rate $\bar{\chi}$. That is, $\zeta_{\mathrm{ms}}^{\prime}$ decreases in the axial direction as the fluid becomes more completely mixed. Values of $\zeta_{\mathrm{ms}}^{\prime}$ are largest if coaxial air with swirl is added. With coaxial air alone, the profile shown in Fig. 6a is narrower than the jet and swirl cases, which indicates that the coaxial air reduces the spreading angle of the shear layer and that mixing is occurring at a relatively inward radial location. This observation is consistent with the fact that coaxial air should reduce the velocity difference across the shear layer. With swirl, the mixing region in Fig. 6 is seen to be broadened by the centrifugal forces present.

\section{Measured Joint PDF $(\zeta, \chi)$}

Several new mixing model $\mathrm{s}^{19}$ have identified the mixture fraction and the dissipation rate as the two most important scalars because the dissipation rate $\chi$ can be related to the local strain rate. ${ }^{11}$ Therefore it

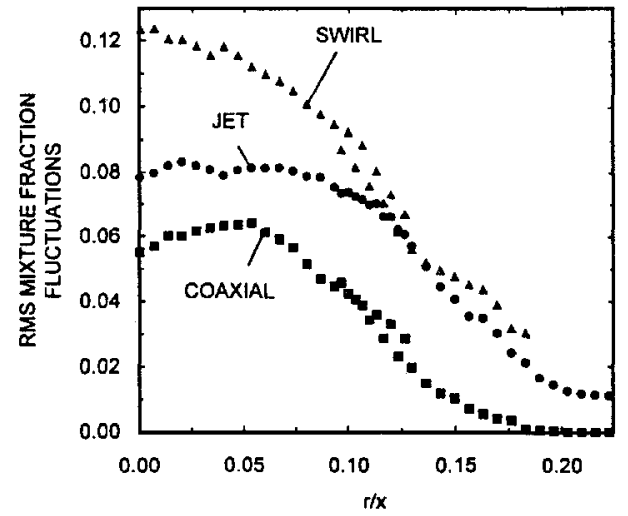

a) $x / d=14$

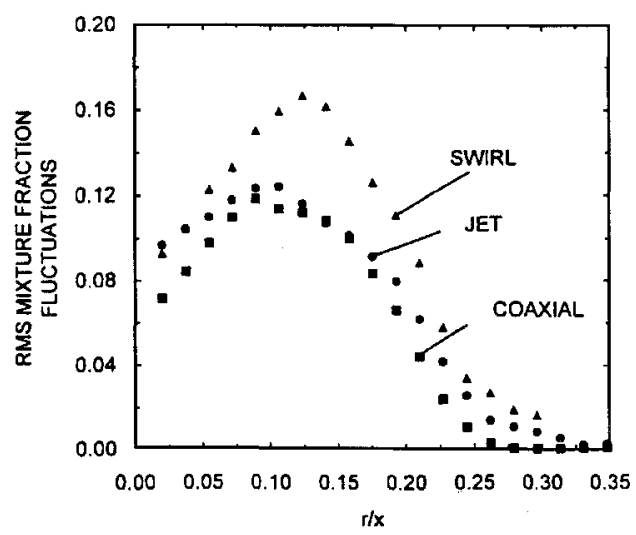

b) $x / d=5$

Fig. 6 RMS fluctuations of the mixture fraction, which indicate the local unmixedness of the flow field. Note that trends are similar to those displayed by the dissipation rate in Fig. 5 . 


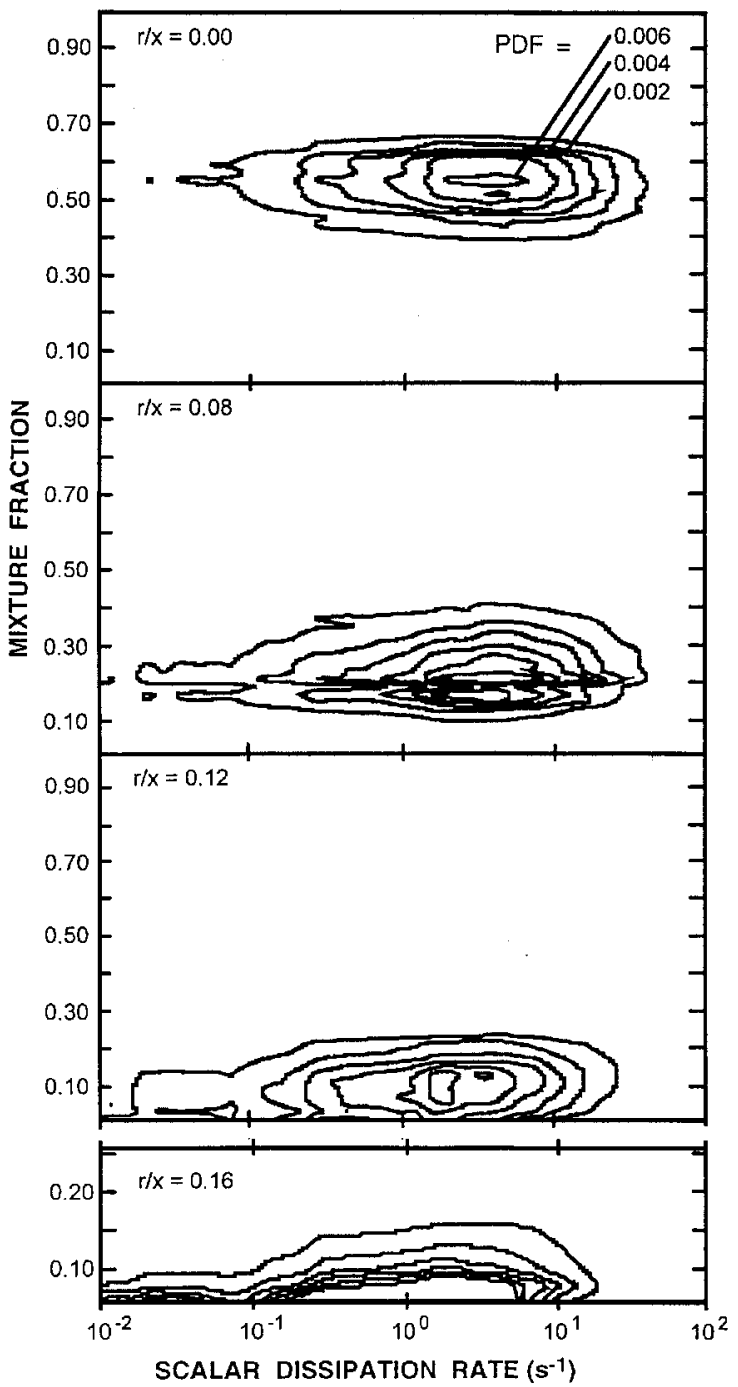

Fig. 7 Measured joint PDF $(\zeta, \chi)$ in the jet at $x / d=14$. Jet case; no coaxial air or swirl. Note that the peak of the PDF moves downward and to the left since mean $\zeta$ and $\chi$ decrease as $r$ increases.

is possible to add the effects of strain to certain models if the shape of the joint PDF is known. In a reacting flow, $\chi$ describes the deviations from chemical equilibrium when a laminar flamelet approach is used. ${ }^{19}$ The shape of the joint PDF $(\zeta, \chi)$ was determined from the present data because it plays a central role in such models.

The joint PDF $(\zeta, \chi)$ that is measured in the jet at $x / d=14$ is shown in Fig. 7. The corresponding shapes of the functions PDF $(\zeta)$ and $\operatorname{PDF}(\chi)$ are shown in Figs. 8 and 9 . Figures $8 \mathrm{a}$ and $8 \mathrm{~b}$ represent the projection on the $\zeta$ axis of the three-dimensional PDF shown in Fig. 7, after integration over all $\chi$ values. It is seen that the upper PDF in Fig. 7 , which was obtained at $r / x=0$, displays considerable symmetry in both the $\zeta$ and $\chi$ directions. This symmetry also is apparent in Figs. $8 \mathrm{a}$ and 9 . However, near the outer boundary of the jet at $r / x=0.16$, Fig. 7 shows that the PDF is no longer symmetric, which also is apparent in Fig. 8b. The joint PDF contours in Fig. 7 move down and to the left as the outer edge of the jet is approached, because the mean values of both $\zeta$ and $\chi$ are decreasing in this direction.

The PDFs of mixture fraction in Fig. 8a show good agreement with the Gaussian curve that is plotted as a solid line. Figure $8 \mathrm{~b}$ indicates that near the outer edge of the jet the PDFs are clipped Gaussians with a second maximum occurring at $\zeta=0$, as has been reported by Namazian et al. ${ }^{3}$ and Dowling and Dimotakis, ${ }^{20}$ among others, and is consistent with temperature PDFs reported by Anselmet and Antonia ${ }^{7}$ in a heated jet and by Everest et al. ${ }^{6}$ in a jet flame. The advantage of the present high-resolution imaging system is that information about the dissipation rate and its PDF can be obtained over a wide range of $\chi$ values, from a lower limit of $0.1 \mathrm{~s}^{-1}$ (which is set by shot noise) to an upper limit of $450 \mathrm{~s}^{-1}$ (which is set by the spatial resolution of $170 \mu$ ).

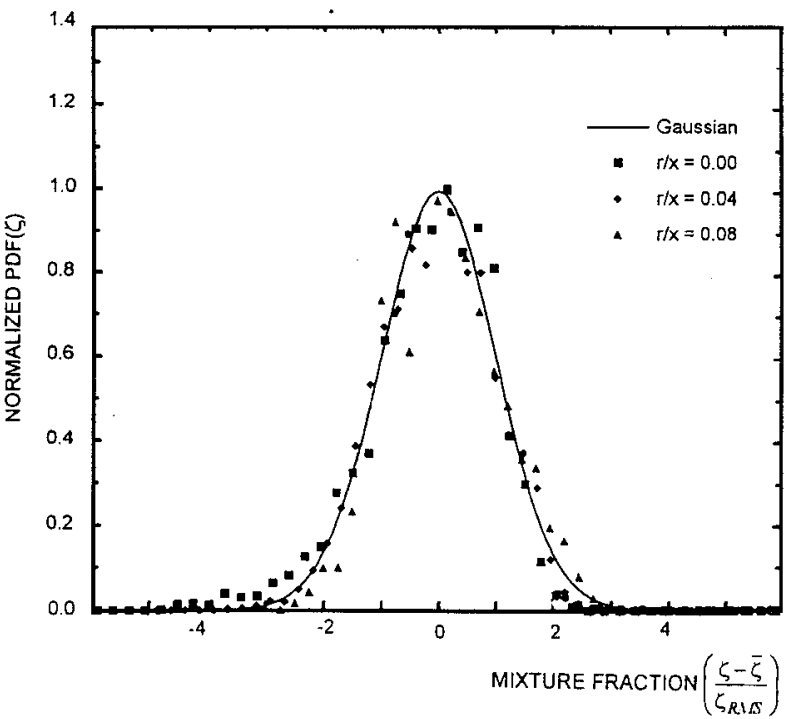

Fig. 8a Comparison of the PDF of mixture fraction to a clipped Gaussian. Jet case, $x / d=14$. Values of mean mixture fraction $\zeta$ are 0.54 , 0.41 , and 0.23 at $r / x=0.0,0.04$, and 0.08 , respectively; rms mixture fraction values are $0.07,0.08$, and 0.08 at $r / x=0.0,0.04$, and 0.08 , respectively.

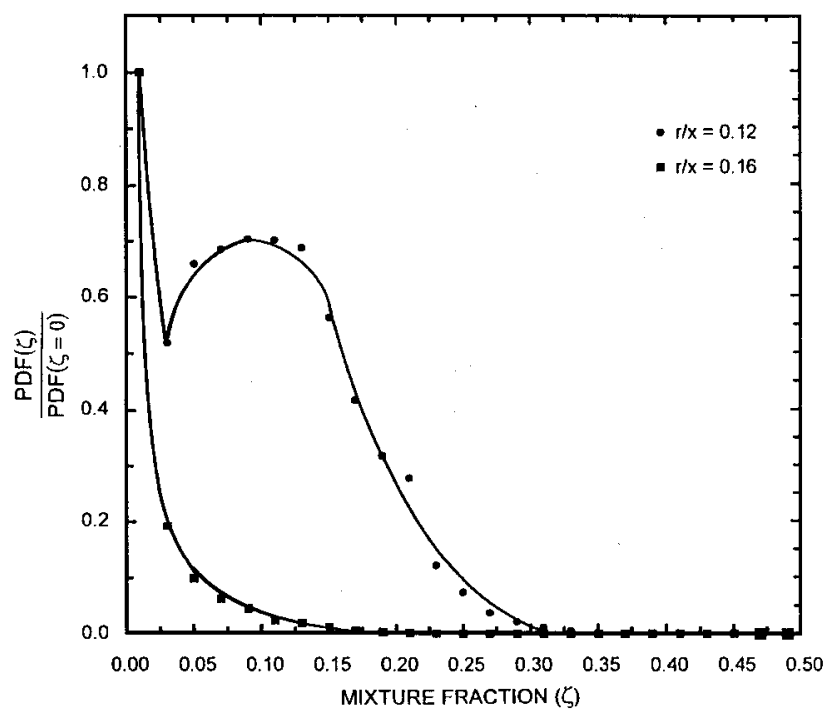

Fig. 8b PDF of mixture fraction near the jet edge. Note that the PDF loses its Gaussian shape due to intermittency.

Figure 9 shows that the PDF of dissipation rates at $x / d=14$ in the jet does display some deviation from a classical log-normal distribution, which is shown as a solid curve. Data are normalized such that the peak value on each curve occurs at the location $(0,1)$. All of the curves in Fig. 9 show good agreement with the log-normal curve on the right-hand side of the figure (where $\chi$ exceeds its most probable value), yet the curves also show large deviations from the log-normal curve for dissipation rates less than the most probable value.

The large probability of small gradients shown in Fig. 9 indicates that in the base region of a jet there are relatively more homogeneous regions present (that are unmixed or fully mixed) than one would expect to find in a fully developed, isotropic turbulent mixing process. The deviations from the log-normal PDF are not surprising because the images presented in Figs. 1b, 1d, 1f, and $1 \mathrm{~h}$ indicate that there are large tongues of entrained air that are associated with small values of $\chi$. The results shown in Fig. 9 imply that mixing models of the base region of a jet should account for large-scale entrainment and nonisotropic dissipation layers and should allow for deviations from a simple log-normal PDF of dissipation rate. One way to account for such deviations is to derive equations to predict a variable PDF shape. ${ }^{21}$ 


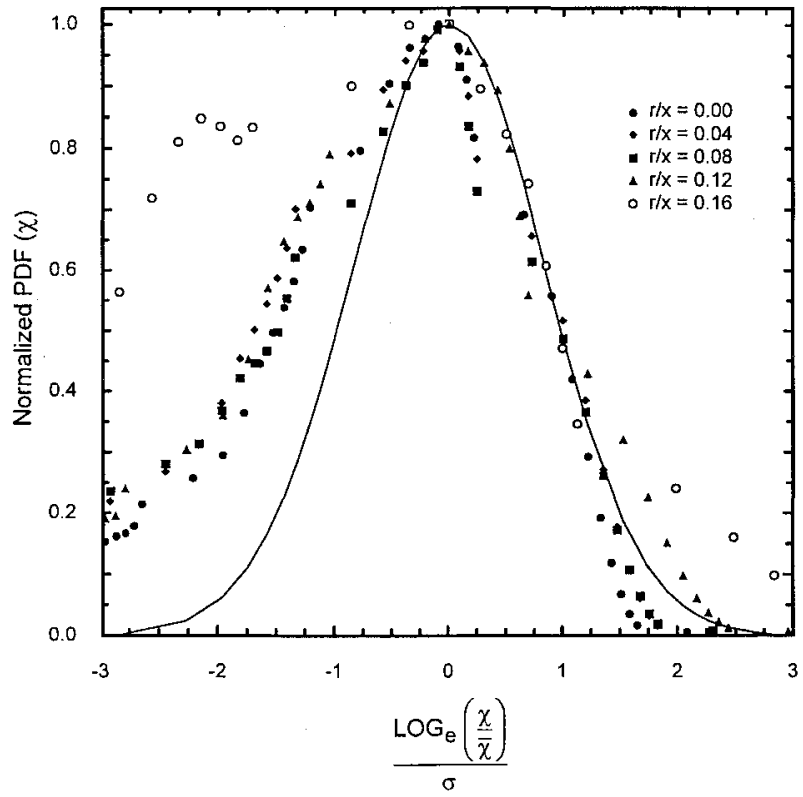

Fig. 9 Comparison of the PDF of scalar dissipation rate to a log-normal distribution. Jet case, $x / d=14$. Mean dissipation rate and standard deviation are $\left(7.0 \mathrm{~s}^{-1}, 1.6 \mathrm{~s}^{-1}\right)$ at $r / x=0.0,\left(7.0 \mathrm{~s}^{-1}, 1.5 \mathrm{~s}^{-1}\right)$ at $r / x=0.08$, and $\left(4.0 \mathrm{~s}^{-1}, 0.8 \mathrm{~s}^{-1}\right)$ at $r / x=0.16$.

\section{Measurement Errors}

Errors arise due to two primary sources for the present measurements: shot noise and spatial resolution limitations. Spatial resolution limitations were discussed earlier. The noise-to-signal ratio associated with shot noise is inversely proportional to the product of the following quantities: laser energy per pulse, the collection solid angle, the Rayleigh scattering cross section, and the area on the image plane over which the signal is averaged. These quantities were optimized by using a high-energy $200-\mathrm{mJ}$ laser pulse, an efficient $/ / 1.2$ optical collection lens, and propane, whose cross section is 13 times larger than that of air. Shot noise-to-signal ratios $(N / S)$ were less than $2 \%$, which is the measured ratio of $\mathrm{rms}$ to mean signal variations in a pure propane region. The error in the mean conserved scalar is $(N / S) /(1+n)^{1 / 2}$, based on the $\chi^{2}$ criterion, where $n$ the number of samples, which is 200 in the present case. The resulting error in the mean conserved scalar due to shot noise is approximately $1 \%$. However, the error in the measurement of gradients is significantly larger; values of $\bar{\chi}$ measured in pure air were $0.1 \mathrm{~s}^{-1}$, which is typically $3 \%$ of the centerline values of $\bar{\chi}$.

\section{Conclusions}

1) In the base region of a jet, the most intense dissipation layers are aligned at approximately $45 \mathrm{deg}$ to the flow, in contrast to the nearly isotropic alignment of layers in the far field reported by Dahm et al. ${ }^{1}$ The observed layer alignment is believed to be due to vortex rotation that entrains air radially inward and in the flow direction; the most intense layers occur at the boundaries of the tongues of entrained air.

2) Profiles of mean scalar dissipation rate, which is a direct measure of the local mixing rate, display a shape that is consistent with the general scaling relation of Peters and Williams, ${ }^{11}$ except that $\bar{\chi}$ does not approach zero on centerline. The constant in the scaling relation for $\bar{\chi}$ was measured to be 9300 . The maximum values of $\bar{\chi}$ in the jet are $14.2 \mathrm{~s}^{-1}$ at $x / d=5$ and $4.9 \mathrm{~s}^{-1}$ at $x / d=14$; these values are larger than those reported by Namazian et al. ${ }^{3}$ and by Effelsberg and Peters ${ }^{18}$ but can be explained by the larger values of the velocity and gas density of the present jet.

3) The addition of coaxial air with swirl caused local fragmentation of the mixture fraction field, many islands of entrained air, and an increase in $\bar{\chi}$ to $30 \mathrm{~s}^{-1}$, which is twice that of the simple jet. The increased value of the dissipation rate was primarily due to an increase of the number of dissipation layers per unit volume and not due to larger values of $\chi$ within each layer.
4) Measurements are reported of the joint $\operatorname{PDF}(\zeta, \chi)$, which is a key element of certain mixing models. In the jet base region $(x / d=$ $5-14)$ the PDF of mixture fraction has a clipped-Gaussian shape, which is characteristic of fully developed turbulence, yet the PDF of the scalar dissipation rate exhibits some deviations from a lognormal distribution. This deviation is not surprising since the images reveal that the dissipation layers are not isotropically oriented in the jet base region.

\section{Acknowledgments}

This research was supported by Gas Research Institute Contract 5087-260-1443, which was monitored by J. Kezerle and R. Serauskas. Discussions with Werner J. A. Dahm and Kenneth Buch assisted the thesis efforts of the first two authors and are appreciated.

\section{References}

${ }^{1}$ Dahm, W. J. A., Southerland, K. B., and Buch, K. A., "Direct, High Resolution Four-Dimensional Measurements of the Fine Scale Structure of Sc $\gg 1$ Molecular Mixing in Turbulent Flows," Physics of Fluids A, Vol. 3 , No. 5, 1991, pp. 1115-1127.

${ }^{2}$ Buch, K. B., Dahm, W. J. A., Dibble, R. W., and Barlow, R. S., "Structure of the Equilibrium Reaction Rate Fields in Turbulent Jet Flames," Proceed ings of the 24th Symposium (International) on Combustion, Combustion Inst., Pittsburgh, PA, 1992, pp. 295-301.

${ }^{3}$ Namazian, M., Schefer, R. W., and Kelly, J., "Scalar Dissipation Measurements in the Developing Region of a Jet," Combustion and Flame, Vol. 74, No. 2, 1988, pp. 147-160.

${ }^{4}$ Schefer, R. W., Kerstein, A. R., Namazian, M., and Kelly, J., "Role of Large Scale Structures in a Nonreacting Turbulent $\mathrm{CH}_{4}$ Jet," Physics of Fluids, Vol. 6, No. 2, 1994, pp. 652-661.

${ }^{5}$ Nandula, S. P., Brown, T. M., and Pitz, R. W., "Measurements of Scalar Dissipation in the Reaction Zones of Turbulent Nonpremixed $\mathrm{H}_{2}$ Air Flames," Combustion and Flame, Vol. 99, No. 3, 1994, pp. 775-780.

${ }^{6}$ Everest, D. A., Driscoll, J. F., Dahm, W. J. A., and Feikema, D. A. "Images of the Temperature Field and Temperature Gradients to Quantify Mixing Rates Within a Non-Premixed Turbulent Jet Flame," Combustion and Flame, Vol. 101, No. 2, 1995, pp. 58-68

7 Anselmet, F., and Antonia, R. A., "Joint Statistics Between Temperature and Its Dissipation in a Turbulent Jet," Physics of Fluids, Vol, 28, No. 4 1985, pp. 1048-1054

${ }^{8}$ Boyer, L. M., and Queiroz, M., "Temperature Dissipation Measurements in a Lifted Turbulent Diffusion Flame," Combustion Science and Technology, Vol. 79, No. 1, 1991, pp. 1-34.

${ }^{9}$ Lockwood, F., and Moneib, H. A., "Fluctuating Temperature Measurements in a Turbulent Jet Diffusion Flame," Combustion Science and Technology, Vol. 22, 1980, pp. 63-81.

${ }^{10}$ Bilger, R. W., "Turbulent Flows with Nonpremixed Reactants," Turbulent Reacting Flows, edited by P. A. Libby and F. A. Williams, Vol. 44, Topics in Applied Physics, Springer-Verlag, New York, 1980, pp. 65-113.

${ }_{11}$ Peters, N., and Williams, F. A., "Liftoff Characteristics of Turbulent Jet Diffusion Flames," AlAA Journal, Vol. 21, No. 3, 1983, pp. 423-429.

${ }^{12}$ Tangirala, V., Chen, R. H., and Driscoll, J. F., "Effect of Heat Release and Swirl on the Recirculation Within Swirl Stabilized Flames," Combustion Science and Technology, Vol. 51, No. 1, 1986, pp. 75-95.

${ }^{13}$ Antonia, R. A., Browne, L. W. B., Chambers, S. J., and Rajagopalan, S., "Budget of the Temperature Variance in a Turbulent Plane Jet," International Journal of Heat and Mass Transfer, Vol. 26, No. 1, 1983, pp. 41-49.

${ }^{14}$ Sreenivasan, K. R., Antonia, R. A., and Dahn, H. Q., "Temperature Dissipation Fluctuations in a Turbulent Boundary Layer," Physics of Fluids, Vol. 20, No. 8, 1977, pp. 1238-1243.

${ }^{15}$ Lim, J. S., Two Dimensional Signal and Image Processing, PrenticeHall, Englewood Cliffs, NJ, 1990.

${ }^{16}$ White, F., Viscous Fluid Flow, 2nd ed., McGraw-Hill, New York, 1991.

${ }^{17}$ Wygnanski, I., and Fiedler, H., "Some Measurements in the SelfPreserving Jet," Journal of Fluid Mechanics, Vol. 38, Pt. 3, 1969, pp. 577612.

${ }^{18}$ Effelsberg, E., and Peters, N., "Scalar Dissipation Rates in Turbulent Jets and Jet Diffusion Flames," Proceedings of the 22nd Symposium (In ternational) on Combustion, Combustion Inst., Pittsburgh, PA, 1989, pp. 345-352.

${ }^{19}$ Smith, N. S. A., Bilger, R. W., and Chen, J. Y., "Modelling of Nonpremixed Hydrogen Jet Flames Using a Conditional Moment Closure Method," Proceedings of the 24th Symposium (International) on Combustion, Combustion Inst., Pittsburgh, PA, 1992, pp. 263-269.

${ }^{20}$ Dowling, D. R., and Dimotakis, P. E., "Similarity of the Concentration Field of Gas-Phase Turbulent Jets," Journal of Fluid Mechanics, Vol. 218, 1990, pp. 109-141.

${ }^{21}$ Chen, J.-Y., Kollman, W., and Dibble, R. W., "Pdf Modelling of Turbulent Methane-Air Nonpremixed Jet Flames," Combustion Science and Technology, Vol. 64, No. 5, 1989, pp. 315-346. 